\title{
Intracardiac Metastasis from Epitheloid Sarcoma: Rare Localization and Difficult Management
}

\author{
Karima Oualla ${ }^{1,2 *}$, Sofia Latifian ${ }^{1}$, Aspasia Georgala ${ }^{1}$, Fabienne Lebrun $^{1}$, François Delhaye ${ }^{3}$, \\ Marc Lemort ${ }^{4}$, Omar Elmesbahi2 ${ }^{2}$, Thierry Gil'1, Ahmad Awada1 \\ ${ }^{1}$ Department of Medical Oncology, Jules Bordet Institute, Brussels, Belgium \\ ${ }^{2}$ Department of Medical Oncology, Hassan II University Hospital, Fez, Morocco \\ ${ }^{3}$ Department of Cardiology, Jules Bordet Institute, Brussels, Belgium \\ ${ }^{4}$ Department of Radiology, Jules Bordet Institute, Brussels, Belgium \\ Email: karimarauf@hotmail.com
}

Received 21 February 2014; revised 20 March 2014; accepted 2 April 2014

Copyright (C) 2014 by authors and Scientific Research Publishing Inc.

This work is licensed under the Creative Commons Attribution International License (CC BY).

http://creativecommons.org/licenses/by/4.0/

(c) (i) Open Access

\section{Abstract}

The involvement of the heart in metastatic cancer is a very rare clinical diagnosis with poor prognosis given to the major risk of cardiac failure. They are frequently asymptomatic or symptoms, when present, may be attributed to other causes. The most common, among the latter, are intrathoracic cancers, lymphomas, leukemias, melanoma, and rarely sarcomas. The echocardiography is the gold standard for diagnosis, but scanner and magnetic resonance imaging (MRI) can be helpful for determination of exact location and composition of lesions. Cardiac metastases occur generally in advanced stage in poly-metastatic patients. Treatment is often in a palliative strategy but should be discussed in multidisciplinary approach for each case. We report a case of cardiac metastasis occurring in a 47 years old woman, treated for epitheloid sarcoma of the buttock. The aim of this work is to show the rarity of the heart location, describing the epidemiological, clinical, radiological, and prognostic features of these metastases and finally discussing the therapeutic strategy.

\section{Keywords}

Heart, Metastasis, Echocardiography, MRI, Chemotherapy, Prognosis

\footnotetext{
${ }^{*}$ Corresponding author.
} 


\section{Introduction}

Cardiac metastases are a very rare entity and generally associated with poor prognosis because of cardiac failure. Although post-mortem studies would suggest that cardiac metastases occur frequently, many of these metastases remain clinically silent or unidentified due to overshadowing by symptoms of the primary malignancy [1]. However, secondary spread to the heart is important to recognize, as it occurs mainly in the context of disseminated disease and portends a poor prognosis [2], as illustrated by our case.

\section{Case Presentation}

We report the case of a 47 years old woman, in her medical history we found anemia, and anal fissure, followed since January 2007 for an epithelioid sarcoma of the left buttock. She was initially treated by wide resection then adjuvant radiotherapy. In July 2012, the patient presented multifocal recurrence in bone, lung and lymph node. She received four cycles of chemotherapy based on doxorubicin and ifosfamide.

After disease progression she received Trabectidine for 3 cycles. The third line of treatment was Dacarbazine.

The patient was admitted in hospital for deterioration of performance statue and management of mandibular pain due to metastatic bone lysis, for which she received radiotherapy as an analgesic therapy. Evaluation after 3 cycles of dacarbazine was performed showing disease progression.

The cardiac control before starting a new line of treatment with Pazopanib was performed because she received several drugs before and especially anthracycline (Doxorubicin) which is known for its cardiac toxicity. We note that patient had essentially major asthenia but evolution was marked by occurrence of dyspnea at the slightest movement. The echocardiography had objectified a mass at the bottom of the left atrium extending to the pulmonary anastomosis, mobile, suggestive of intracardiac metastasis (Figure 1). The left ventricular ejection fraction was conserved. The same observation had been reported at the transesophageal ultrasound.

Cardiac MRI confirmed the presence of a tumor process in the left atrium in continuity with the infiltration of the right superior pulmonary vein with suspected infiltration of the right atrium and infiltration of multiple pulmonary arterial branches. Radiologist confirmed that the aspect is very characteristic of heart's metastasis, excluding other differential diagnosis especially primary (benign or malignant) tumors (Figure 2(a) and Figure 2(b)).

Biopsy for histological confirmation was decided but finally not performed given to the clinical deterioration of patient and the characteristic metastatic aspect on the echocardiography and cardiac MRI. Resection of the cardiac process and radiation therapy were discussed and rejected because it is locally advanced and invades vessels and also given to the character multi-metastatic of the disease with poor prognosis and compromised survival. Anticoagulation was started in order to reduce the thrombo-embolic risk. After 2 months of Pazopanib, evolution has been a marked by clinical and radiological progression. At this moment clinical symptoms of cardiac failure had clearly appeared. Treatment was stopped and we decide to give supportive care only. A control by echocardiography showed the progression of cardiac mass that filled the entire left atrium.

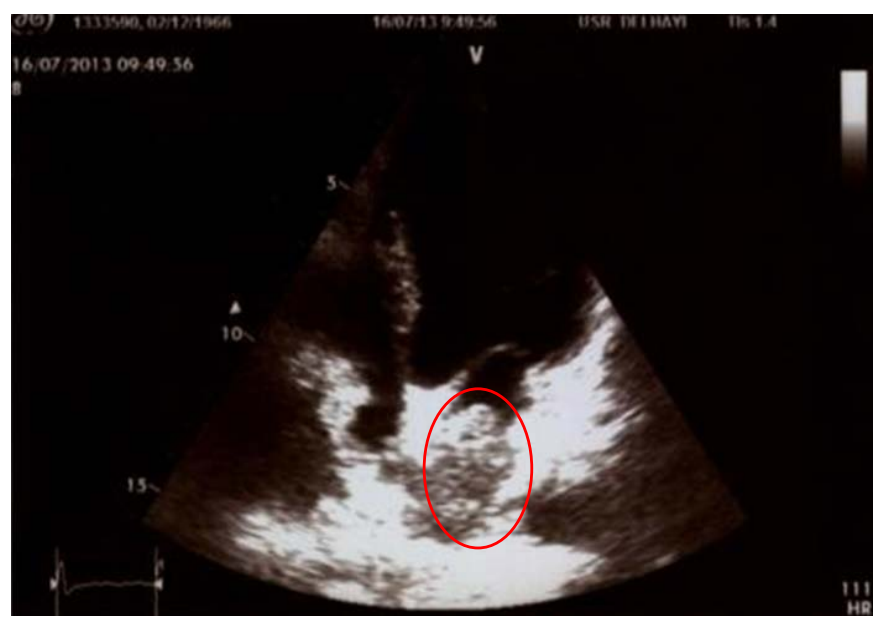

Figure 1. Trans-thoracic echocardiography showing an intra-cardiac metastasis at the left atrium. 


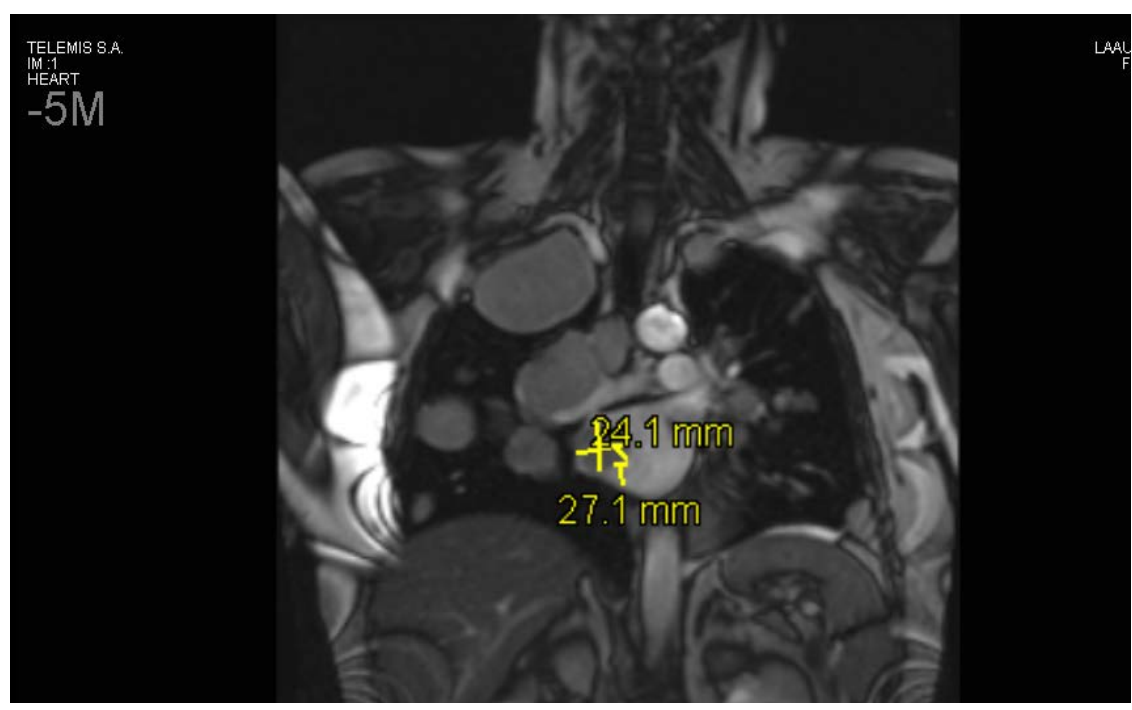

(a)

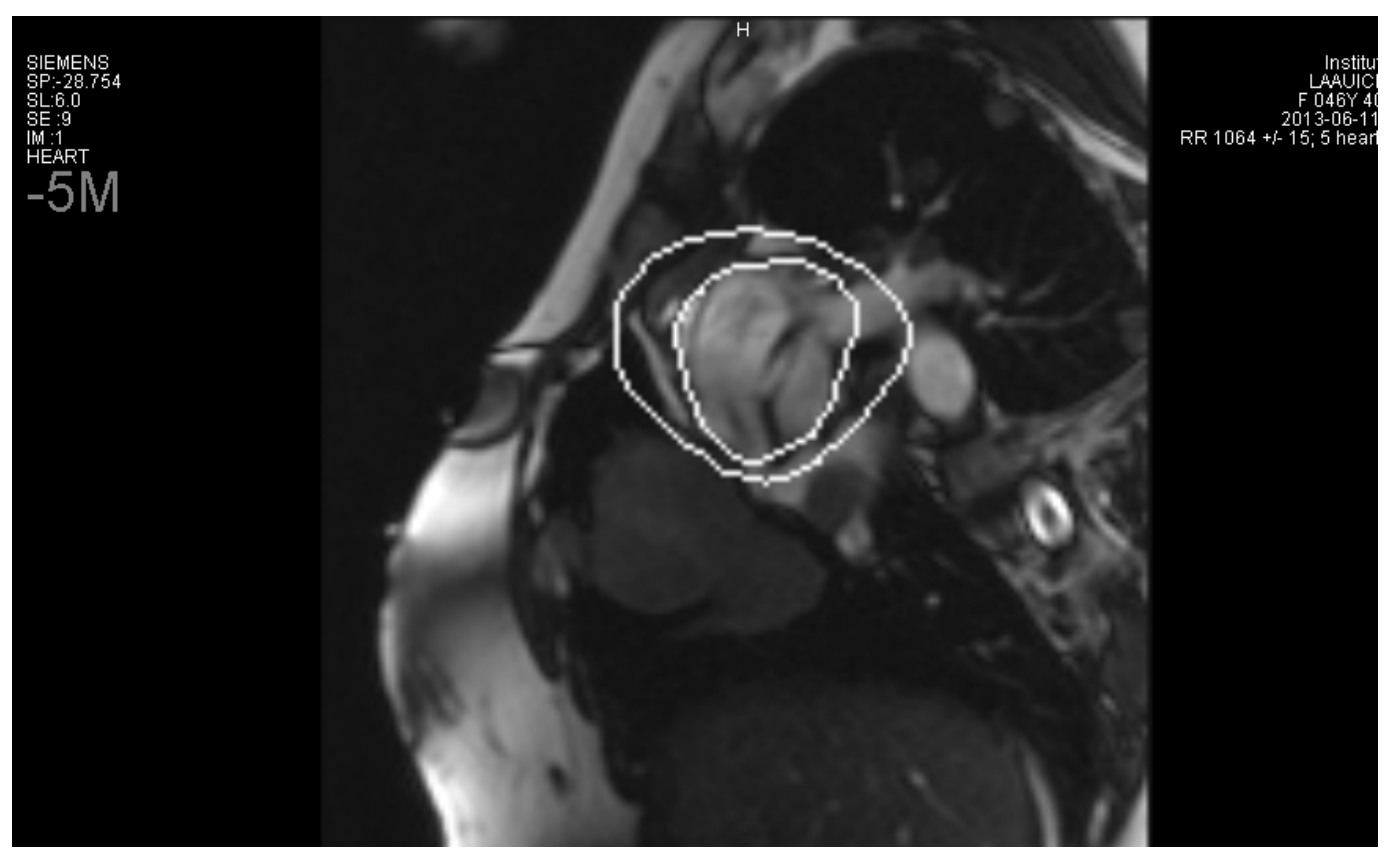

(b)

Figure 2. Cardiac MRI showing intra-cardiac metastasis at the bottom of left atrium atrium extending to the pulmonary anastomosis.

\section{Discussion}

Heart is an uncommon site of metastases. Although post-mortem studies would suggest that cardiac metastases occur frequently, but many of these metastases remain clinically silent.

The most common, among the latter, are lung, breast and oesophageal cancers, lymphomas, leukemias and finally melanoma, which has the highest rate of cardiac metastasis [3]. Based on data from postmortem studies of Bussani et al. during the period from 1994 to 2003, the incidence of cardiac metastases involves 9.1\% of early-stage cancers and $14.2 \%$ of metastatic. The most common sites of origin include the lung (pleural mesothelioma $48.4 \%$, adenocarcinoma $21 \%$, squamous-cell carcinoma 18.2\%), skin (melanoma 27.8\%), breast (15.5\%) and undifferentiated carcinomas (19.5\%) [1].

Hallahan et al. [4] reported that cardiac metastases were present in $25 \%$ of consecutive autopsies of patients 
with soft-tissue sarcoma, which is higher than recognized clinically, suggesting that most cases are probably missed. Cardiac metastasis often causes immediate death of a patient, so establishment of appropriate management is very important. However, little is known in terms of its precise clinical features.

By location, pericardial metastases are more common than myocardial or endocardial metastases. Approximately two thirds of cardiac metastases involve the pericardium, and one third resides in the myocardium or/ and the epicardium, with only $5 \%$ of cases involving the endocardium. This distribution is determined by the different ways in which cancer is spreading [2]. Pericardial infiltration is either a result of local direct invasion by intrathoracic tumors or derives from myocardial involvement and furthermore through retrograde lymphatic spreading from mesothoracic and tracheal nodes. Myocardial or epicardial metastasis is usually hematogenous or lymphogenous whereas the endocardium is generally invaded through systematic circulation and in fewer cases through myocardial involvement [1] [2]. Cardiac metastases are usually small and multiple; however, single large tumor lesions are also observed. In carcinomas, metastatic deposits may lead to diffuse thickening of the pericardium. Focal as well as diffuse tumor infiltration have been observed in hematological malignancies: in contrast to leukemic infiltration, lymphomatous deposits are usually grossly discernible. Although it is assumed that the right side of the heart is more frequently involved than the left [5] [6] there are numerous cases where left-sided involvement is found [7] [8].

The clinical presentations are diverse according to the location of metastasis, but in general cardiac metastases are silent or pauci-symptomatic, only at $10 \%$, particularly in patients with advanced disease [3]. Pericardial involvement may cause dyspnea, hypotension, tachycardia and even signs of cardiac tamponade due to pericardial effusion. Myocardial infiltration is associated with arrhythmias and if generalized it may be responsible for congestive heart failure and systolic or diastolic dysfunction [9]. Rarely, a neoplastic thrombus in the coronary circulation can cause an acute myocardial infarction through invasion, or strangulation of coronary arteries by a massive pericardial effusion. When the metastasis is in the intra-ventricular location, the obstruction may damage the tricuspid or mitral valve function or spread neoplastic emboli to the arterial circulation of the lungs after being ruptured. Moreover, the organization of neoplastic thrombi inside the right atrium or ventricule may facilitate the dispersal of cancer cells to distant metastatic sites.

The involvement of the right-side of heart is more common than the left-sided. It can be explained by systolic pressure and lower right flow. Conversely, invasion to the heart valves is rare because of constituent movement and lack of vascularization [9].

The clinical presentation in our patient was silent and didn't guide to the diagnosis of cardiac metastasis.

Conduction disorders, atrial or ventricular arrhythmias or ST-segment abnormalities can be seen at the electrocardiogram. Regarding the chest $\mathrm{x}$-rays; it may show enlarged cardiac silhouette and pleural effusion as common findings. But the gold standard for the diagnosis of cardiac metastases is the two-dimensional echocardiography; it often shows pericardial thickening or fluid, mobility abnormalities of myocardial wall and intraventricular masses [2]. The second type of echocardiography which is trans-esophageal ultrasound is also helpful especially for diagnosis of peri and para-cardial metastases. The computed tomography and magnetic resonance imaging have a complementary role to the trans-thoracic echocardiography, they provide sections of cardiac, mediastinal, pulmonary and thoracic structures in any desired plane, without overlapping, they give more precision in determination of exact location and composition of lesions [9].

Concerning the role of positron emission tomography (PET) scan, Johnson and al reported that its expanding use, will increase the number of diagnosed cases [10].

In our patient, in accordance with literature, the trans-thoracic echocardiography had revealed the diagnosis of cardiac metastasis, then confirmed by trans-esophageal echography. The MRI had confirmed the diagnosis and had given more information about location and composition of lesion.

When the primary tumor was resected many years before detection of a cardiac tumor mass, exploratory thoracotomy and open biopsy might be necessary to confirm the diagnosis of metastasis [11].

Given the character poly-metastatic of the disease at the moment of diagnosis of cardiac metastases, the management of patients remains in a palliative strategy. Because at this stage of the disease, many patients will already have undergone surgical treatment for the tumor of origin, or radio- or chemotherapy, Cardiac treatment is mostly confined to palliative measures. Surgical resection is only indicated for solitary lesions causing obstruction of intra-ventricular or valvular flow in patients of good prognosis with total resection of the primary tumour [1] [12]. 
Cardiac metastases can decrease when responding well to radio- or chemotherapy, especially for in leukemia and lymphoma [13] [14]. Pericardial effusion can also be managed with local radiotherapy or systemic chemotherapy. Another option is the local infusion of chemotherapeutic agents (Ex: bleomycin, cis-platin, mitomycin C, 5-FU) or radioisotopes [15].

However, improvement of symptoms is not frequent because of cardiopulmonary side effects, like fibrosis of the lung or myocardium, pericarditis, and conduction troubles for radiotherapy. Cardiotoxic side effects of some chemotherapeutic agents like anthracyclines and when used with high doses may induce recalcitrant myocardial failure.

Surgical resection is only indicated in exceptional cases of unique metastasis, leading to obliteration of cardiac chambers or valve obstruction if the tumor of origin was surgically resected in totality and the prognosis seems to be good.

Our patient illustrated the case of cardiac metastasis occurring at an advanced stage of disease with a polymetastatic cancer for which a new line of palliative treatment was given but the disease had rapidly progressed then only supportive care was confined to the patient.

\section{Conclusion}

Cardiac metastases are very rare and have a poor prognosis as they usually occur in patients with multi-metastatic disease and given the major risk of heart failure. The diagnosis is often difficult and delayed because of rarity and misleading character of symptoms. Echocardiography is the standard for the diagnosis. More precision is obtained by the scanner and especially cardiac MRI. In general, treatment is in palliative approach because the advanced stage of the disease; but a multidisciplinary team including medical oncologist, radiologist, cardiologist, pathologist, surgeon and radiotherapist is mandatory for optimal treatment. Cardiac metastasis should be considered when cardiac symptoms are present despite their rarity.

\section{Acknowledgements}

I acknowledge anyone who contributed in the elaboration of this work. This work received no specific grant from any funding structure.

\section{Competing Interest}

Authors have declared that no competing interests exist.

\section{Authors' Contribution}

All authors had participated to the study, each one in his field, and they all read and approved the final manuscript.

\section{Consent}

Patient was agreed and had consent to participate to this work.

\section{References}

[1] Bussani, R., De-Giorgio, F., Abbate, A. and Silvestri, F. (2007) Cardiac Metastases. Journal of Clinical Pathology, 60, 27-34. http://dx.doi.org/10.1136/jcp.2005.035105

[2] Reynen, K., Kockeritz, U. and Strasses, R.H. (2004) Metastases to the Heart. Annals of Oncology, 15, 375-381. http://dx.doi.org/10.1093/annonc/mdh086

[3] Roberts, W.C. (1997) Primary and Secondary Neoplasms of the Heart. American Journal of Cardiology, 80, 671-682. http://dx.doi.org/10.1016/S0002-9149(97)00587-0

[4] Hallahan, D.E., Vogelzang, N.J., Borow, K.M., Bostwick, D.G. and Simon, M.A. (1986) Cardiac Metastases from Soft-Tissue Sarcomas. Journal of Clinical Oncology, 4, 1662-1669.

[5] Prichard, R.W. (1951) Tumors of the Heart. Review of the Subject and Report of One Hundred and Fifty Cases. Archives of Pathology, 51, 98-128. 
[6] Glancy, D.L. and Roberts, W.C. (1968) The Heart in Malignant Melanoma. A Study of 70 Autopsy Cases. American Journal of Cardiology, 21, 555-571. http://dx.doi.org/10.1016/0002-9149(68)90289-0

[7] MacGee, W. (1991) Metastatic and Invasive Tumours Involving the Heart in a Geriatric Population: A Necropsy Study. Virchows Arch A, 419, 183-189. http://dx.doi.org/10.1007/BF01626346

[8] Scott, R.W. and Garvin, C.F. (1939) Tumors of the Heart and Pericardium. American Heart Journal, 17, 431-436. http://dx.doi.org/10.1016/S0002-8703(39)90593-4

[9] Hoey, E.T., Mankad, K., Puppala, S., Gopalan, D. and Sivananthan, M.U. (2009) MRI and CT Appearances of Cardiac Tumours in Adults. Clinical Radiology, 64, 1214-1230. http://dx.doi.org/10.1016/j.crad.2009.09.002

[10] Johnson, T.R., Becker, C.R., Wintersperger, B.J., Herzog, P., Lenhard, M.S. and Reiser, M.F. (2005) Images in Cardiovascular Medicine. Detection of Cardiac Metastasis by Positron-Emission Tomography-Computed Tomography. Circulation, 112, e61-e62.

[11] Janssen, D.P.B., Van de Kaa, C.A., Noyez, L., et al. (1994) A Solitary Metastasis in the Heart from Ewing’s Sarcoma. European Journal Cardio-Thoracic Surgery, 8, 51-53. http://dx.doi.org/10.1016/1010-7940(94)90135-X

[12] Vlachostergiosa, P.J., Daliania, D.D. and Papandreoua, C.N. (2012) Basic Concepts in Metastatic Cardiac Disease. Cardiology Research, 3, 47-48.

[13] Cham, W.C., Freiman, A.H., Carstens, P.H.B. and Chu, F.C.H. (1975) Radiation Therapy of Cardiac and Pericardial Metastases. Radiology, 114, 701-704.

[14] Hanfling, S.M. (1960) Metastatic Cancer to the Heart. Review of the Literature and Report of 127 Cases. Circulation, 22, 474-483. http://dx.doi.org/10.1161/01.CIR.22.3.474

[15] Al-Mamgani, A., Baartman, L., Baaijens, M., de Pree, I., Incrocci, L. and Levendag, P.C. (2008) Cardiac Metastases. International Journal of Clinical Oncology, 13, 369-372. http://dx.doi.org/10.1007/s10147-007-0749-8 